\title{
Different dimensional fractional-order discrete chaotic systems based on the Caputo $h$-difference discrete operator: dynamics, control, and synchronization
}

\author{
Ibtissem Talbi' , Adel Ouannas², Amina-Aicha Khennaoui ${ }^{3}$ Abdelhak Berkane1, lqbal M. Batiha4, \\ Giuseppe Grassi ${ }^{5}$ and Viet-Thanh Pham ${ }^{6 *}$
}

\author{
${ }^{*}$ Correspondence: \\ phamvietthanh@tdtu.edu.vn \\ ${ }^{6}$ Nonlinear Systems and \\ Applications, Faculty of Electrical \\ and Electronics Engineering, Ton \\ Duc Thang University, Ho Chi Minh \\ City, Vietnam \\ Full list of author information is \\ available at the end of the article
}

\begin{abstract}
The paper investigates control and synchronization of fractional-order maps described by the Caputo $h$-difference operator. At first, two new fractional maps are introduced, i.e., the Two-Dimensional Fractional-order Lorenz Discrete System (2D-FoLDS) and Three-Dimensional Fractional-order Wang Discrete System (3D-FoWDS). Then, some novel theorems based on the Lyapunov approach are proved, with the aim of controlling and synchronizing the map dynamics. In particular, a new hybrid scheme is proposed, which enables synchronization to be achieved between a master system based on a 2D-FoLDS and a slave system based on a 3D-FoWDS. Simulation results are reported to highlight the effectiveness of the conceived approach.
\end{abstract}

Keywords: Discrete fractional calculus; Control; Synchronization; Discrete Lorenz system; Discrete Wang system; Lyapunov approach

\section{黛 Springer}

\section{Introduction}

The primary interpretation of the discrete fractional calculus (DFC), which is being deemed one of the great modern branches in the field of calculus, was first proposed by Diaz and Olserin in 1974 [1]. Over the previous decade, several results in theories and applications of such a branch have been carried out, with the aim to provide mathematical models for a number of natural phenomena. Examples include the use of DFC for modeling the movement of a bead on a wire [2], as well as for describing optimal control systems [3].

As a matter of fact, several types of difference discrete operators (DDOs), which are frequently proposed in the DFC field, could be derived and formulated through discretizing their corresponding continuous operators as in [1]. These operators are related to various fractional-order derivatives (FoDs), such as the Riemann-Liouville (RL) derivative [4, 5], the Caputo derivative [6], and the Grünwald-Letnikov (GL) derivative [7]. In particular, these three types of operators fall under the name of fractional $h$-DDOs [8]. Further de-

C) The Author(s) 2020. This article is licensed under a Creative Commons Attribution 4.0 International License, which permits use, sharing, adaptation, distribution and reproduction in any medium or format, as long as you give appropriate credit to the original author(s) and the source, provide a link to the Creative Commons licence, and indicate if changes were made. The images or other third party material in this article are included in the article's Creative Commons licence, unless indicated otherwise in a credit line to the material. If material is not included in the article's Creative Commons licence and your intended use is not permitted by statutory regulation or exceeds the permitted use, you will need to obtain permission directly from the copyright holder. To view a copy of this licence, visit http://creativecommons.org/licenses/by/4.0/. 
velopments of another operator, called $h$-sum operator, have been recently accomplished in [8]. Moreover, some discrete chaotic systems have been explored in accordance with the so-called v-Caputo delta DDO (see [9-11]). Additionally, in [12] different fractional difference operators have been studied and formulated on the isolated time scale with step $h$. In particular, difference operators with different discrete kernels have been studied in [12], including the power law, the exponential law, and the Mittag-Leffler law. In [13] fractional difference operators with discrete generalized Mittag-Leffler kernels have been illustrated for both the Atangana-Baleanu-Riemann type and the Atangana-BaleanuCaputo type. In [14] two types of dual identities for Caputo fractional differences are investigated. Namely, the first type relates nabla and delta type fractional sums and differences, whereas the second type (represented by the $Q$-operator) relates left and right fractional sums and differences [14]. In [15] two types of dual identities for Riemann fractional sums and differences have been investigated, along with the solution for a higher-order Riemann fractional difference equation. In [16] the stability of discrete nonautonomous systems (in the sense of the Caputo fractional difference) has been studied via the Lyapunov direct method. In particular, the conditions for uniform stability, uniform asymptotic stability, and global uniform asymptotic stability for such systems have been deeply analyzed $[16,17]$. Despite all these studies, so far an accurate definition of the fractional-order DDO (FoDDO) has not been agreed upon. Namely, what has been agreed upon is the fact that such an operator has infinite memory (unlike the integer operator [18]) and represents an extension of the binomial formulation via the Gamma function [19].

Over the last few years, several fractional-order difference models have been discretized based on efficient tools introduced by the DFC. The so-called fractional-order chaotic discrete systems (FoCDSs) are the most significant among those models [19]. The first examples of chaotic maps have been presented in $[20,21]$. In particular, in [20] a fractional logistic map is proposed and its chaotic behavior is numerically illustrated. In [21] a fractional Lorenz map is introduced and its chaotic synchronization is studied based on the stability results. References [20] and [21] have received significant consideration by many researchers, also due to the wide range of applications of chaotic maps in applied science and engineering areas. In particular, the infinite memory characteristic of fractional maps allows flexible modeling, along with the capability to achieve higher degree of chaotic behaviors. These features actually increase the usefulness of chaotic maps in several applications, including data encryption [22, 23], secure communications [24-27], and control theory [28]. Typical examples of such chaotic discrete-time systems include the Lozi map [29], the Hénon map [30], the generalized Hénon map [31], the Stefanski map [32], the Baier-Klein system [33], the Rossler map [34], the Lorenz system [35], and the Wang map [36]. It has been become apparent that these maps have richer dynamics compared to the classical ones [19]. In other words, their trajectories highlight very complex dynamic behaviors, depending on both the initial conditions and the fractional-order values $[18,37]$. Referring to the very recent results in the literature regarding fractional discrete-time systems, a new fractional logistic map with two parameters is proposed in [38], along with an image encryption application. In [39] a novel short-memory fractional modeling approach is applied to memristors, neural networks, and material's relaxation property. In [40] new variable-order fractional chaotic systems are proposed. In particular, the fractional order is defined by the use of a piecewise constant function, which leads to rich chaotic dynamics, for both continuous- and discrete-time systems [40]. 
In general, when dealing with FoCDSs, two main aspects should be explored, i.e., control and synchronization of their chaotic modes [37]. Controlling these systems consists in proposing a suitable adaptive controller for their chaotic modes, so that their states are forced to be asymptotically stable, or are stabilized at zero [18, 41, 42]. Control issues are, for instance, of great importance in several industrial processes, like in robotics where chaotic motions of a rigid body need to be controlled [43-45]. On the other hand, synchronization, which has been considered a key concept in chaos theory over the last three decades, targets to compel the states of a slave system to tend towards the exact trajectories that are determined by a master system, assuming that both systems start from different initial points in phase space [19]. Different synchronization and control techniques have been suggested and implemented on some FoCDSs [18, 19, 35, 46]. One could observe that all the aforementioned works, that have discussed both issues of synchronization and control, have employed some linearization methods or some nonlinear laws to implement their strategies [47]. As far as we know, the topic of controlling and synchronizing FoCDSs based on $h$-DDOs remains, to date, a new and almost unexplored field.

Based on these considerations, this paper makes a contribution to the topic of FoCDSs by presenting novel versions of two- and tree-dimensional Lorenz and Wang fractional chaotic maps, respectively, as well as by providing efficient improvements in the schemes for controlling and synchronizing their dynamics. This objective is achieved by introducing novel theorems that exploit Lyapunov-based approaches [48, 49]. The paper is organized as follows. Section 2 introduces the definition of fractional $h$-DDOs and useful results related to the Lyapunov stability. In Sect. 3 some new versions of FoDCSs are introduced via the Caputo $h$-DDO, and their chaotic dynamics are analyzed in detail. In Sect. 4 linear control laws are proposed to stabilize the dynamics of the considered FoDCSs at the origin. In Sect. 5 a new hybrid scheme is proposed, which enables synchronization to be achieved between a master system based on the two-dimensional fractional Lorenz map and a slave system based on the three-dimensional fractional Wang map. Finally, simulation results are reported to highlight the effectiveness of the conceived approach.

\section{Fractional $h$-DDOs and Lyapunov stability}

As already mentioned, the DFC is considered a relatively new topic that has not been settled, yet. From this perspective, this section presents some preliminaries and notations related to such topic for completeness.

Definition $1([5])$ Let $X:(h \mathbb{N})_{a} \rightarrow \mathbb{R}$. For a given $v>0$, the $v$ th-order $h$-sum is given by

$$
{ }_{h} \Delta_{a}^{-v} X(t)=\frac{h}{\Gamma(v)} \sum_{s=\frac{a}{h}}^{\frac{t}{h}-v}(t-\sigma(s h))^{(v-1)} x(s h), \quad \sigma(s h)=(s+1) h, t \in(h \mathbb{N})_{a+v h},
$$

where $a \in \mathbb{R}$ is a starting point and the $h$-falling factorial function is defined as

$$
t_{h}^{(v)}=h^{v} \frac{\Gamma\left(\frac{t}{h}+1\right)}{\Gamma\left(\frac{t}{h}+1-v\right)}, \quad t, v \in \mathbb{R}
$$

while $(h \mathbb{N})_{a+(1-v) h}=\{a+(1-v) h, a+(2-v) h, \ldots\}$. 
Definition $2([6,12])$ For a function $x(t)$ defined on $(h \mathbb{N})_{a}$, and for a given $v>0$ such that $v \notin \mathbb{N}$, the Caputo $h$-DDO is defined by

$$
{ }_{h}^{C} \Delta_{a}^{v} X(t)=\Delta_{a}^{-(n-v)} \Delta^{n} X(t), \quad t \in(h \mathbb{N})_{a+(n-v) h},
$$

where $\Delta X(t)=\frac{X(t+h)-X(t)}{h}$ and $n=\lceil\nu\rceil+1$.

Remark 1 Using the Caputo $h$-difference operator is useful when dealing with applications of control theory. Namely, controllability (i.e., the possibility to transfer the considered system from a given initial state to a final state using controls from some set) and observability (i.e., the possibility of reconstruction of an initial state using control inputs and output sequences) are both readily achievable when a fractional discrete system is described via the Caputo $h$-difference operator [50,51]. Examples of the usefulness in adopting the Caputo $h$-difference operator are illustrated in [52-54], regarding the controllability and observability of fractional control systems.

From the point of view of obtaining a significant result and a useful inequality for Lyapunov functions reported in [48], which are briefly illustrated below, some stability conditions of the zero equilibrium point for a nonlinear fractional-order difference discrete system will be identified later on. Such a nonlinear system has the form:

$$
{ }_{h}^{C} \Delta_{a}^{v} X(t)=f(t+v h, X(t+v h)), \quad t \in(h \mathbb{N})_{a+(1-v) h} .
$$

Theorem 1 ([48]) Let $x=0$ be an equilibrium point of system (3). If there exists a positive definite and decreasing scalar function $V(t, X(t))$ such that ${ }_{h}^{C} \Delta_{a}^{v} V(t, X(t)) \leq 0$, then the equilibrium point is asymptotically stable.

Lemma 2 ([48]) For any discrete time $t \in(h \mathbb{N})_{a+(1-v) h}$, the following inequality holds:

$$
{ }_{h}^{C} \Delta_{a}^{v} X^{2}(t) \leq 2 X(t+v h)_{h}^{C} \Delta_{a}^{v} X(t)
$$

where $0<v \leq 1$.

\section{Some new forms of FoDCSs}

In this part, two new forms of the FoDCSs are introduced using fractional $h$-DDOs. The first is associated with the Two-Dimensional Fractional-order Lorenz Discrete System (2D-FoLDS), while the second one is associated with the Three-Dimensional Fractionalorder Wang Discrete System (3D-FoWDS).

\subsection{D-FoLDS}

The earlier release of the FoLDS was established in [55] using the $v$-Caputo delta DDO. It turned out that this map, which possesses two nonlinear terms, is actually chaotic for some proper values of its parameters $(\alpha, \beta)$ and fractional-orders $v$, where $v \in(0,1]$. Herein, a new version of $2 \mathrm{D}$-FoLDS is derived using, this time, the Caputo $h$-DDO. In particular, the following equations are proposed:

$$
\left\{\begin{array}{l}
{ }_{h}^{C} \Delta_{a}^{v} x_{m}(t)=\alpha \beta x(t+v h)-\beta y(t+v h) x(t+v h), \\
{ }_{h}^{C} \Delta_{a}^{v} y_{m}(t)=\beta\left(x(t+v h)^{2}-y(t+v h)\right),
\end{array}\right.
$$


where ${ }_{h}^{C} \Delta_{a}^{v}$ denotes the Caputo $h$-DDO, $t \in(h \mathbb{N})_{a+(1-v) h}, a \in \mathbb{R}$ is the starting point, and $\alpha$ and $\beta$ are the system's parameters. Map (5), however, can be regarded as a generalized form of the FoLDS constructed in [55]. Its solution, moreover, can be obtained via employing the fractional $h$-sum operator. That is, the two corresponding implicit discrete formulae of the two equations given in (5) are reported in [8] as follows:

$$
\left\{\begin{array}{l}
x(n)=x(0)+\frac{h^{v}}{\Gamma(v)} \sum_{j=1}^{n} \frac{\Gamma(n-j+v)}{\Gamma(n-j+1)}(\alpha \beta x(j)-\beta y(j) x(j)), \\
y(n)=y(0)+\frac{h^{v}}{\Gamma(v)} \sum_{j=1}^{n} \frac{\Gamma(n-j+v)}{\Gamma(n-j+1)}\left(\beta\left(x^{2}(j)-y(j)\right)\right),
\end{array}\right.
$$

subject to the given initial conditions $x(0)$ and $y(0)$.

In the light of the predictor-corrector scheme (see [56]), the two equations given in (6) could be converted into another two explicit forms which might then be utilized for examining the dynamic behavior of system (5). Anyhow, taking the initial conditions $x(0)=$ 0.2 and $y(0)=0.3$, the fractional-order value $v=0.9$, and the system's parameters $\alpha=1$ and $\beta=0.73$ yields the attractor of map (5) exhibited in Fig. 1(a). Furthermore, Fig. 1(b)-(c) shows the resultant calculations of both the bifurcation diagram and the largest Lyapunov exponent (LLE) as a function of $\alpha$. Obviously, the chaotic behavior of system (5) has been demonstrated in those figures according when $\alpha=0.95, \beta=1$, and $\nu=0.9$.

\subsection{D-FoDWS}

The first form of the FoDWS, with its classical case due to Wang, was addressed and explored well by considering also the $v$-Caputo DDO in [37]. Like the previously proposed map, the Caputo $h$-DDO is employed to propose the following new 3D-FoDWS:

$$
\left\{\begin{array}{l}
{ }_{h}^{C} \Delta_{a}^{v} x(t)=\alpha_{3} y(t+v h)+\alpha_{4} x(t+v h), \\
{ }_{h}^{C} \Delta_{a}^{v} y(t)=\alpha_{1} x(t+v h)+\alpha_{2} z(t+v h), \\
{ }_{h}^{C} \Delta_{a}^{v} z(t)=\alpha_{7} z(t+v h)+\alpha_{6} y(t+v h) z(t+v h)+\alpha_{5},
\end{array}\right.
$$

where $t \in(h \mathbb{N})_{a+(1-v) h}$ and $\alpha_{i}$ 's are the system's parameters, $i=1,2, \ldots, 7$. Accordingly, the three equations given in (7) have the following equivalent numerical formulae:

$$
\left\{\begin{array}{l}
x(n)=x(0)+\frac{h^{v}}{\Gamma(v)} \sum_{j=1}^{n} \frac{\Gamma(n-j+v)}{\Gamma(n-j+1)}\left(\alpha_{3} y(j)-\alpha_{4} x(j)\right), \\
y(n)=y(0)+\frac{h^{v}}{\Gamma(v)} \sum_{j=1}^{n} \frac{\Gamma(n-j+v)}{\Gamma(n-j+1)}\left(\alpha_{1} x(j)+\alpha_{2} z(j)\right), \\
z(n)=z(0)+\frac{h^{v}}{\Gamma(v)} \sum_{j=1}^{n} \frac{\Gamma(n-j+v)}{\Gamma(n-j+1)}\left(\alpha_{7} z(j)+\alpha_{6} y(j) z(j)+\alpha_{5}\right) .
\end{array}\right.
$$

In Fig. 2(b), the attractor of map ( 7$)$ is displayed by considering the initial conditions $x(0)=$ $0.5, y(0)=0.6, z(0)=0.02$, and assuming $v=0.9$, whereas the system's parameters are set to be $\left(\alpha_{1}, \alpha_{2}, \alpha_{3}, \alpha_{4}, \alpha_{5}, \alpha_{6}, \alpha_{7}\right)=(-1.9,0.2,0.5,-2.3,2,-0.6,-1.9)$. Furthermore, the resultant calculation of the bifurcation diagram as a function of $\alpha_{3}$ is exhibited in Fig. 2(a). Thence, it has been clearly shown that the chaotic behavior of system (7) will occur, e.g., when $h=0.1, v=0.9$, and when the same values of $\alpha_{i}$ 's are taken as above, where $i=1,2, \ldots, 7$.

\section{Linear control laws}

This section proposes two control laws related to the 2D-FoLDS and 3D-FoWDS. Besides, the Lyapunov approach is employed to establish the asymptotic convergence of the two controllers. 
$(a)$

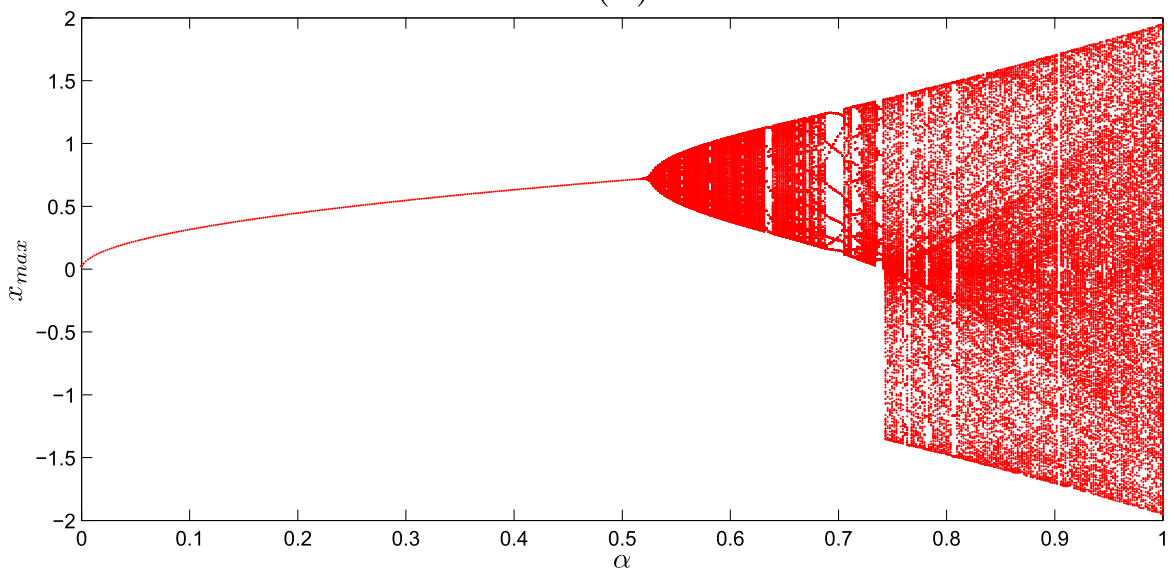

(b)

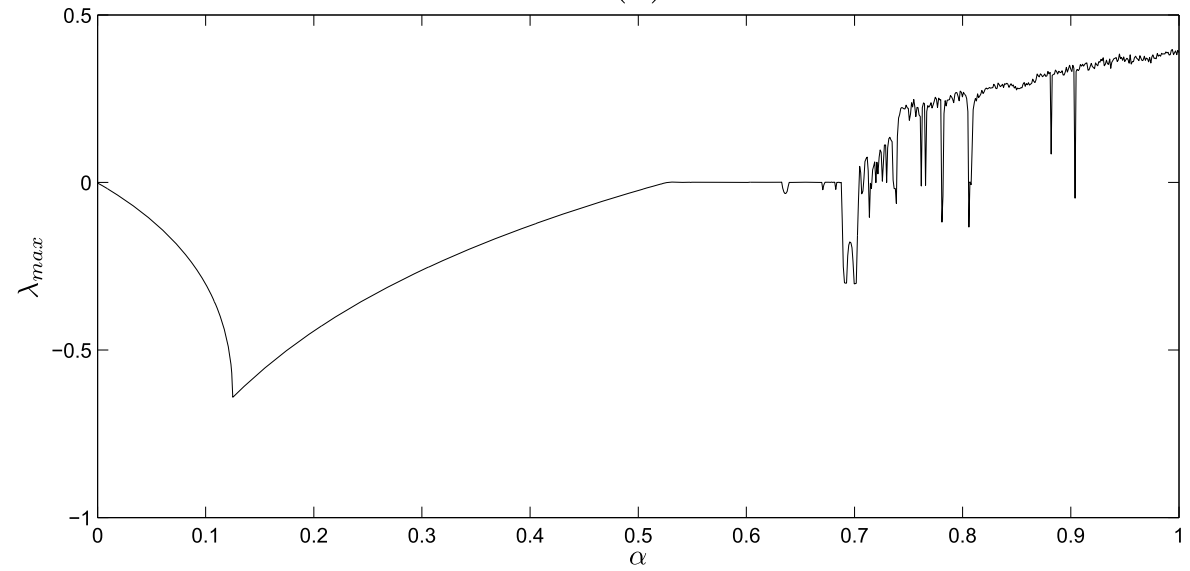

(c)

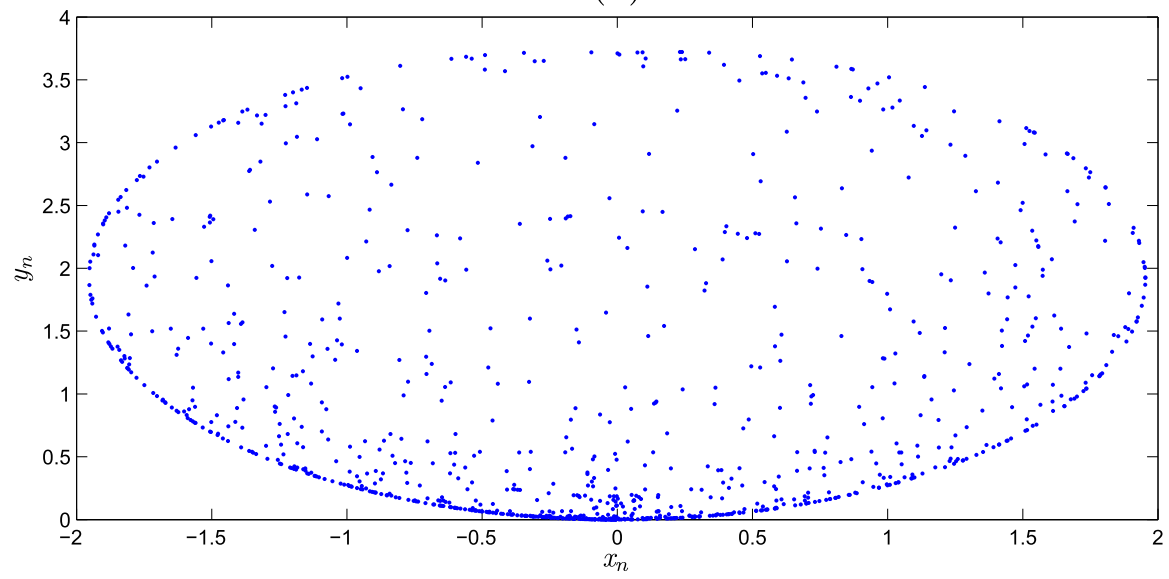

Figure 1 Bifurcation and the LLE diagrams of the 2D-FoLDS versus $\alpha$, for system's parameter $\beta=0.73$ and initial condition $x_{0}=0.2, y_{0}=0.3$ : (a) bifurcation diagram and (b) LLE. (c) Chaotic attractor of the 2D-FoLDS for $\alpha=1, \beta=0.75$ and $\nu=0.9$ 
(a)
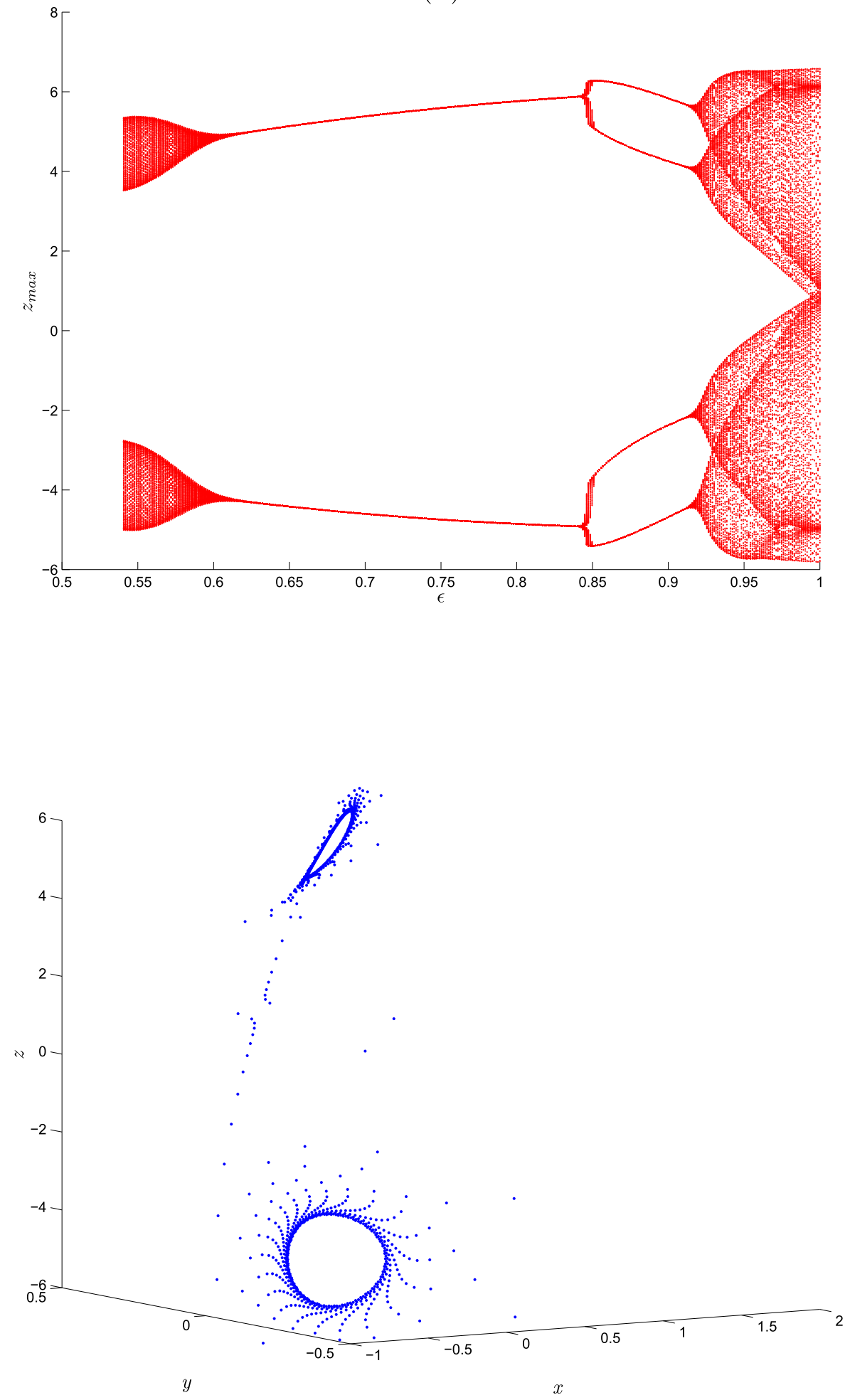

Figure 2 Bifurcation diagram and chaotic attractor of the 3D-FoWDS versus $\alpha_{3}$, for system's parameter $\left(\alpha_{1}, \alpha_{2}, \alpha_{3}, \alpha_{4}, \alpha_{5}, \alpha_{6}, \alpha_{7}\right)=(-1.9,0.2,0.5,-2.3,2,-0.6,-1.9)$ and initial condition $x_{0}=0.5, y_{0}=0.6, z_{0}=0.02$ : (a) bifurcation diagram and (b) chaotic attractor 
Theorem 3 The 2D-FoLDS given in (5) can be controlled under the following onedimensional control law:

$$
\mathbf{C}(t)=-(1+\alpha \beta) x(t), \quad t \in(h \mathbb{N})_{a+(1-v) h} .
$$

Proof Considering (5) yields its controlled version, of course, under the controller given in (9). This version has the form

$$
\left\{\begin{array}{l}
{ }_{h}^{C} \Delta_{a}^{v} x(t)=\alpha \beta x(t+v h)-\beta y(t+v h) x(t+v h)+\mathbf{C}(t+v h), \\
{ }_{h}^{C} \Delta_{a}^{v} y(t)=\beta\left(x(t+v h)^{2}-y(t+v h)\right) .
\end{array}\right.
$$

Consequently, (10) takes the form

$$
\left\{\begin{array}{l}
{ }_{h}^{C} \Delta_{a}^{v} x(t)=-x(t+v h)-\beta y(t+v h) x(t+v h), \\
{ }_{h}^{C} \Delta_{a}^{v} y(t)=\beta\left(x(t+v h)^{2}-y(t+v h)\right) .
\end{array}\right.
$$

One might employ the Lyapunov approach by first considering the Lyapunov function, $V(t)$, in the form:

$$
V=\frac{1}{2} x^{2}(t)+\frac{1}{2} y^{2}(t)
$$

The adoption of the Caputo $h$-DDO yields

$$
{ }_{h}^{C} \Delta_{a}^{v} V(t)=\frac{1}{2}{ }_{h}^{C} \Delta_{a}^{v} x^{2}(t)+\frac{1}{2}{ }_{h}^{C} \Delta_{a}^{v} y^{2}(t) .
$$

Using Lemma 2 leads to the following steps:

$$
\begin{aligned}
{ }_{h}^{C} \Delta_{a}^{v} V \leq & x(t+v h)_{h}^{C} \Delta_{a}^{v} x(t)+y(t+v h)_{h}^{C} \Delta_{a}^{v} y(t) \\
= & -x^{2}(t+v h)-\beta y(t+v h) x^{2}(t+v h) \\
& +\beta y(t+v h) x(t+v h)^{2}-\beta y^{2}(t+v h) \\
= & \left.-x^{2}(t+v h)-\beta y^{2}(t+v h)<0 \quad \text { (because } \beta=0.73\right) .
\end{aligned}
$$

Hence, it can be deduced, based on Theorem 1, that the equilibrium point at zero of system (11) is asymptotically stable. Therefore, it has been indeed shown that the dynamics of the proposed 2D-FoLDS given in (5) can be stabilized by controller (9).

In order to highlight the potency of the proposed controller, we illustrate the evolution of all the states and the phase-space plots of the controlled system (10) in Fig. 3 when $\alpha=1$, $\beta=0.73$, and $v=0.9$. Obviously, all these plots clearly reflect that the proposed 2D-FoLDS has been completely controlled. Next, an additional control law related to the 3D-FoWDS given in (7) is, moreover, established in identical fashion of the preceding discussion. 

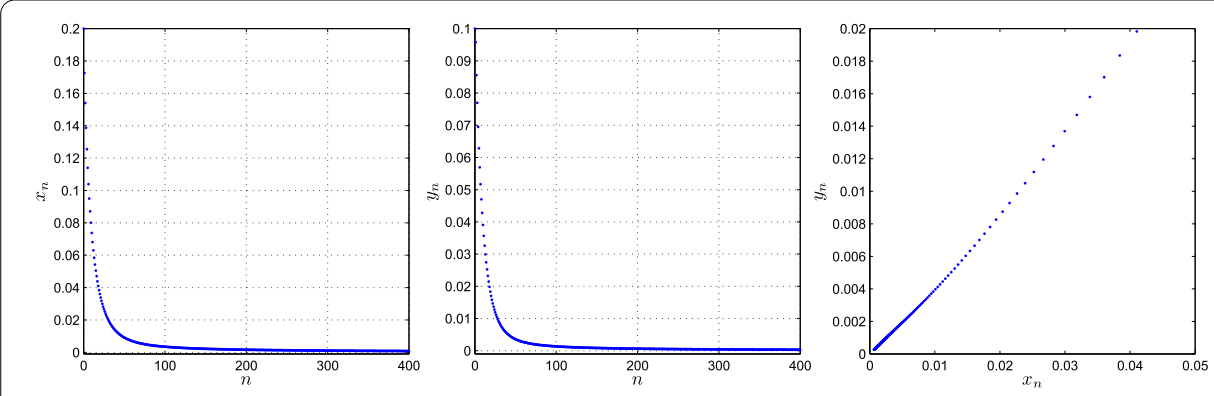

Figure 3 The states and phase space of the controlled 2D-FoLDS

Theorem 4 The 3D-FoWDS given in (7) can be controlled under the following twodimensional control law:

$$
\left\{\begin{array}{l}
\mathbf{L}_{1}(t)=-\left(\alpha_{3}+\alpha_{1}\right) y(t)-\left(\alpha_{4}+1\right) x(t), \\
\mathbf{L}_{2}(t)=-\alpha_{5}-\left(\left|\alpha_{6} b\right|\right) z(t)-\alpha_{2} y(t)
\end{array}\right.
$$

where $|y(t)| \leq b$ and $t \in(h \mathbb{N})_{a+(1-v) h}$.

Proof By considering both (7) and (14), the following controlled map will be deduced:

$$
\left\{\begin{array}{l}
{ }_{h}^{C} \Delta_{a}^{v} x(t)=\alpha_{3} y(t+v h)+\alpha_{4} x(t+v h)+\mathbf{L}_{1}, \\
{ }_{h}^{C} \Delta_{a}^{v} y(t)=\alpha_{1} x(t+v h)+\alpha_{2} z(t+v h), \\
{ }_{h}^{C} \Delta_{a}^{v} z(t)=\alpha_{7} z(t+v h)+\alpha_{6} y(t+v h) z(t+v h)+\alpha_{5}+\mathbf{L}_{2} .
\end{array}\right.
$$

Substituting (14) into (15) yields the following system:

$$
\left\{\begin{array}{l}
{ }_{h}^{C} \Delta_{a}^{v} x(t)=-\alpha_{1} y(t+v h)-x(t+v h), \\
{ }_{h}^{C} \Delta_{a}^{v} y(t)=\alpha_{1} x(t+v h)+\alpha_{2} z(t+v h), \\
{ }_{h}^{C} \Delta_{a}^{v} z(t)=\left(\alpha_{7}-\left|\alpha_{6}\right| b\right) z(t+v h)+\alpha_{6} y(t+v h) z(t+v h)-\alpha_{2} y(t+v h) .
\end{array}\right.
$$

Now, assume the Lyapunov function has the form

$$
V=\frac{1}{2} x^{2}(t)+\frac{1}{2} y^{2}(t)+\frac{1}{2} z^{2}(t)
$$

This implies ${ }_{h}^{C} \Delta_{a}^{v} V={ }_{h}^{C} \Delta_{a}^{v} x^{2}(t)+{ }_{h}^{C} \Delta_{a}^{v} y^{2}(t)+{ }_{h}^{C} \Delta_{a}^{v} z^{2}(t)$, and then by applying Lemma 2, one obtains

$$
\begin{aligned}
{ }_{h}^{C} \Delta_{a}^{v} V \leq & x(t+v h)_{h}^{C} \Delta_{a}^{v} x(t+v h)+y(t+v h)_{h}^{C} \Delta_{a}^{v} y(t)+z(t+v h)_{h}^{C} \Delta_{a}^{v} z(t) \\
= & -\alpha_{1} x(t+v h) y(t-1+v)-x^{2}(t+v h)+\alpha_{1} y(t-1+v) x(t+v h) \\
& +\alpha_{2} y(t+v h) z(t+v h)+\left(\alpha_{7}-\left|\alpha_{6}\right| b\right) z^{2}(t+v h)+\alpha_{6} y(t+v h) z^{2}(t+v h) \\
& -\alpha_{2} z(t+v h) y(t+v h) \\
\leq & -x^{2}(t+v h)+\left(\alpha_{7}-\left|\alpha_{6}\right| b\right) z^{2}(t+v h)+\alpha_{6} y(t+v h) z^{2}(t+v h)
\end{aligned}
$$



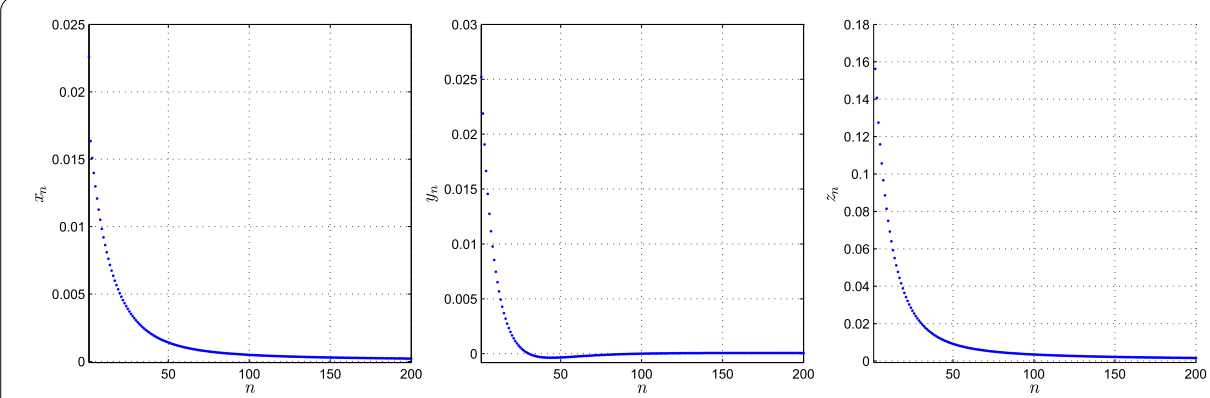

Figure 4 The states of the controlled 3D-FoWDS

$$
\begin{aligned}
& \leq-x^{2}(t+v h)+\left(\alpha_{7}-\left|\alpha_{6}\right| b\right) z^{2}(t+v h)+\left|\alpha_{6} y(t+v h)\right| z^{2}(t+v h) \\
& \leq-x^{2}(t+v h)+\left(\alpha_{7}-\left|\alpha_{6}\right| b\right) z^{2}(t+v h)+\left|\alpha_{6}\right| b z^{2}(t+v h) \\
& \left.=-x^{2}(t+v h)+\alpha_{7} z^{2}(t+v h)<0 \quad \text { (because } \alpha_{7}=-1.9\right) .
\end{aligned}
$$

Again, it can be concluded, based also on Theorem 1, that the equilibrium point at zero of system (16) is asymptotically stable. In this case as well, it has been shown that the dynamics of the other proposed 3D-FoWDS given in (7) could be stabilized by controller (14).

Remark 2 The existence of the upper-bound constant $b$, identified in Theorem 4, is justified by the boundedness property that characterizes all chaotic maps' states.

With the aim of showing some findings associated with Theorem 4, a numerical simulation has been illustrated in Fig. 4. In this figure, the evolution of all the states and the phase-space plots of the controlled system (15) have been exhibited when $v=0.9$ and $\left(\alpha_{1}, \alpha_{2}, \alpha_{3}, \alpha_{4}, \alpha_{5}, \alpha_{6}, \alpha_{7}\right)=(-1.9,0.2,0.5,-2.3,2,-0.6,-1.9)$. Based on such simulation, one can definitely observe that the 3D-FoWDS has been also completely controlled.

Remark 3 Observe that the two controllers established in this section demand a minor control effort due to their linearity.

\section{Hybrid synchronization scheme}

In this part, the two fractional-order maps (2D-FoLDS \& 3D-FoWDS) will be investigated, despite their various dimensions, for the possibility to be synchronized via a suitable synchronization scheme within a certain time. One might suppose the 2D-FoLDS as a master system, and indicate to its states by the subscript, $m$, for each of them. That is,

$$
\left\{\begin{array}{l}
{ }_{h}^{C} \Delta_{a}^{v} x_{m}(t)=\alpha \beta x_{m}(t+v h)-\beta y_{m}(t+v h) x_{m}(t+v h), \\
{ }_{h}^{C} \Delta_{a}^{v} y_{m}(t)=\beta\left(x_{m}(t+v h)^{2}-y_{m}(t+v h)\right) .
\end{array}\right.
$$


At the same time, the 3D-FoWDS is supposed to be a slave system and all its states are indicated by another subscript, say $s$, for each of them, i.e.,

$$
\left\{\begin{array}{l}
{ }_{h}^{C} \Delta_{a}^{v} x_{s}(t)=\alpha_{3} y_{s}(t+v h)+\alpha_{4} x_{s}(t+v h)+\mathbf{U}_{1}, \\
{ }_{h}^{C} \Delta_{a}^{v} y_{s}(t)=\alpha_{1} x_{s}(t+v h)+\alpha_{2} z_{s}(t+v h)+\mathbf{U}_{2}, \\
{ }_{h}^{C} \Delta_{a}^{v} z_{s}(t)=\alpha_{7} z_{s}(t+v h)+\alpha_{6} y_{s}(t+v h) z_{s}(t+v h)+\alpha_{5}+\mathbf{U}_{3},
\end{array}\right.
$$

where $\mathbf{U}_{1}, \mathbf{U}_{2}$, and $\mathbf{U}_{3}$ are the synchronization controllers that need to be established. Actually, the process of picking up an adaptive control law $\left(\mathbf{U}_{1}, \mathbf{U}_{2}, \mathbf{U}_{3}\right)^{T}$ aims to compel the following synchronization errors:

$$
\left\{\begin{array}{l}
e_{1}=x_{s}-x_{m} \\
e_{2}=y_{s}+y_{m} \\
e_{3}=z_{s}-\left(x_{m}+y_{m}\right)
\end{array}\right.
$$

to be asymptotically close to the origin, i.e.,

$$
\lim _{t \rightarrow+\infty}\left|e_{i}(t)\right|=0, \quad \text { for } i=1,2,3 .
$$

Remark 4 In light of the error system (20), it is apparent that the two states $x_{s}$ and $x_{m}$ are completely synchronized, while the state $y_{s}$ is antisynchronized with its corresponding state $y_{m}$, and finally, the state $z_{s}$ appears full-state synchronized with two other states, $x_{m}$ and $y_{m}$. Such three types of synchronization (complete, anti-, and full-state synchronization) show coexisting between the master and slave systems given in (18) and (19), respectively.

For highlighting other significant results related to the proposed synchronization scheme, we state the following theorem which is considered one of the main results of this work.

Theorem 5 The two master and slave systems given in (18) and (19), respectively, achieve synchronized dynamics under the following control law:

$$
\left\{\begin{aligned}
\mathbf{U}_{1}(t)= & -\beta y_{m}(t) x_{m}(t)-\alpha_{3} y_{s}(t)+\left(\alpha \beta-\alpha_{4}\right) x_{m}(t), \\
\mathbf{U}_{2}(t)= & -\beta x_{m}^{2}(t)-\alpha_{2} z_{s}(t)-\alpha_{1} x_{s}(t)-\beta y_{s}(t), \\
\mathbf{U}_{3}(t)= & -\left(\alpha_{7}+1\right) z_{s}(t)-\alpha_{6} y_{s}(t) z_{s}(t)-\beta y_{m}(t) x_{m}(t)-\alpha_{5}+\beta x_{m}^{2}(t) \\
& +(\beta+1) y_{m}(t)+(\alpha \beta+1) x_{m}(t),
\end{aligned}\right.
$$

where $t \in(h \mathbb{N})_{a+(1-v) h}$.

Proof For the purpose of establishing asymptotic convergence of the synchronization errors given in (20) to zero according to (22), we start applying the Caputo $h$-DDO on (20), 
which yields:

$$
\left\{\begin{aligned}
{ }_{h}^{C} \Delta_{a}^{v} e_{1}= & \alpha_{3} y_{s}(t+v h)+\alpha_{4} x_{s}(t+v h)-\alpha \beta x_{m}(t+v h) \\
& +\beta y_{m}(t+v h) x_{m}(t+v h)+\mathbf{U}_{1}, \\
{ }_{h}^{C} \Delta_{a}^{v} e_{2}= & \alpha_{1} x_{s}(t+v h)+\alpha_{2} z_{s}(t+v h)+\beta x_{m}^{2}(t+v h)-\beta y_{m}(t+v h)+\mathbf{U}_{2}, \\
{ }_{h}^{C} \Delta_{a}^{v} e_{3}= & \alpha_{7} z_{s}(t+v h)+\alpha_{6} y_{s}(t+v h) z_{s}(t+v h)+\alpha_{5}-\alpha \beta x_{m}(t+v h) \\
& +\beta y_{m}(t+v h) x_{m}(t+v h)-\beta x_{m}^{2}(t+v h)-\beta y_{m}(t+v h)+\mathbf{U}_{3} .
\end{aligned}\right.
$$

Substituting the proposed control law given in (22) into (23) leads to the following new discrete system:

$$
\left\{\begin{array}{l}
{ }_{h}^{C} \Delta_{a}^{v} e_{1}=\alpha_{4} e_{1}, \\
{ }_{h}^{C} \Delta_{a}^{v} e_{2}=-\beta e_{2}, \\
{ }_{h}^{C} \Delta_{a}^{v} e_{3}=-e_{3} .
\end{array}\right.
$$

Now, letting $V=\frac{1}{2} e_{1}^{2}(t)+\frac{1}{2} e_{2}^{2}(t)+\frac{1}{2} e_{3}^{2}(t)$ implies ${ }_{h}^{C} \Delta_{a}^{v} V={ }_{h}^{C} \Delta_{a}^{v} e_{1}^{2}(t)+{ }_{h}^{C} \Delta_{a}^{v} e_{2}^{2}(t)+{ }_{h}^{C} \Delta_{a}^{v} e_{3}^{2}(t)$, and, by using Lemma 2 , we obtain

$$
\begin{aligned}
{ }_{h}^{C} \Delta_{a}^{v} V & \leq e_{1}(t+v h)_{h}^{C} \Delta_{a}^{v} e_{1}(t+v h)+e_{2}(t+v h)_{h}^{C} \Delta_{a}^{v} e_{2}^{2}(t)+e_{3}(t+v h)_{h}^{C} \Delta_{a}^{v} e_{3}(t) \\
& =\alpha_{4} e_{1}^{2}-\beta e_{2}^{2}-e_{3}^{2}<0 .
\end{aligned}
$$

In the light of Theorem 1, it can be deduced that the dynamics of the error system (20) have been stabilized at the origin. As a consequence, the master and slave systems given in (18) and (19), respectively, have achieved the synchronized dynamics via non-control laws.

In order to show the effectiveness of the proposed approach, Fig. 5 displays the synchronization errors. These plots clearly show that the two fractional-order maps achieve hybrid synchronization.

\section{Conclusion and future work}

This work has established two new versions of the Factional-order Discrete Chaotic Systems (FoDCSs), namely the Two-Dimensional Fractional-order Lorenz Discrete System (2D-FoLDS) and Three-Dimensional Fractional-order Wang Discrete System (2DFoWDS). Using the Caputo $h$-Difference Discrete Operator ( $h$-DDO), all the states of such two versions have been demonstrated to contain chaos. Despite all this, these states could still be controlled through quite simple linear controllers as is demonstrated in some parts of this work. Besides, we have constructed a suitable synchronization scheme which has allowed us to establish a proper controller that has the ability to synchronize the two fractional-order maps under consideration. It has been further shown that all the trajectories of such two maps, together with their proposed controller, converge asymptotically to zero using Lyapunov approach. Finally, several numerical simulations have been performed to highlight the potency of all proposed theoretical findings. 




All the results of this work, without doubt, will inspire us to go deeper into this subject by focusing on two essential steps. The first is related to further experimental implementations of such two maps with the aim of reaching the highest degree of their complexity for their trajectories, whereas the second revolves around the execution of the proposed linear controllers to the field of image encryption. In other words, our future strategy can be described by entering and implementing the proposed master-slave synchronization scheme in a hardware device. This contribution, together with an appropriate encryption algorithm, will allow us to experimentally generate and recover the secret keys.

\section{Acknowledgements}

Adel Ouannas thanks the Directorate General for Scientific Research and Technological Development in Algeria who supported the research at hand.

Funding

None.

Availability of data and materials

Not applicable.

Competing interests

The authors declare that they have no competing interests.

Authors' contributions

Conceptualization, AO, A-AK, IT and IMB; Data curation, GG, AB, AO and V-TP; Investigation, GG, IT, V-TP and IMB:

Methodology, AO; Supervision, V-TP; Validation, AO, GG. All authors read and approved the final manuscript.

\section{Author details}

'Department of Mathematics, University of Constantine, Constantine, Algeria. ${ }^{2}$ Department of Mathematics and Computer Science, University of Larbi Ben M'hidi, Oum El Bouaghi, Algeria. ${ }^{3}$ Laboratory of Dynamical Systems and 
Control, University of Larbi Ben M'hidi, Oum El Bouaghi, Algeria. ${ }^{4}$ Department of Mathematics, Faculty of Science, University of Jordan, Amman, Jordan. ${ }^{5}$ Dipartimento Ingegneria Innovazione, Universita del Salento, 73100, Lecce, Italy. ${ }^{6}$ Nonlinear Systems and Applications, Faculty of Electrical and Electronics Engineering, Ton Duc Thang University, Ho Chi Minh City, Vietnam.

\section{Publisher's Note}

Springer Nature remains neutral with regard to jurisdictional claims in published maps and institutional affiliations.

Received: 26 June 2020 Accepted: 26 October 2020 Published online: 07 November 2020

\section{References}

1. Diaz, J., Osler, T.: Differences of fractional order. Math. Comput. 28(125), 185-202 (1974)

2. Baleanu, D., Jajarmi, A., Asad, J.H., Blaszczyk, T.: The motion of a bead sliding on a wire in fractional sense (2017)

3. Jajarmi, A., Hajipour, M., Mohammadzadeh, E., Baleanu, D.: A new approach for the nonlinear fractional optimal control problems with external persistent disturbances. J. Franklin Inst. 355(9), 3938-3967 (2018)

4. Atici, F.M., Eloe, P.W.: A transform method in discrete fractional calculus. Int. J. Difference Equ. 2(2), 165-176 (2007)

5. Atici, F., Eloe, P.: Initial value problems in discrete fractional calculus. Proc. Am. Math. Soc. 137(3), 981-989 (2009)

6. Abdeljawad, T.: On Riemann and Caputo fractional differences. Comput. Math. Appl. 62(3), 1602-1611 (2011)

7. Ostalczyk, P.: The non-integer difference of the discrete-time function and its application to the control system synthesis. Int. J. Syst. Sci. 31(12), 1551-1561 (2000)

8. Mozyrska, D., Girejko, E.: Overview of fractional h-difference operators. In: Advances in Harmonic Analysis and Operator Theory, pp. 253-268. Springer, Berlin (2013)

9. Wu, G.-C., Baleanu, D.: Discrete fractional logistic map and its chaos. Nonlinear Dyn. 75(1-2), 283-287 (2014)

10. Hu, T.: Discrete chaos in fractional Hénon map. Appl. Math. 2014, 2243-2248 (2014)

11. Shukla, M.K., Sharma, B.B.: Investigation of chaos in fractional order generalized hyperchaotic Henon map. AEÜ, Int. J. Electron. Commun. 78, 265-273 (2017)

12. Abdeljawad, T.: Different type kernel $h$-fractional differences and their fractional $h$-sums. Chaos Solitons Fractals 116 , 146-156 (2018)

13. Abdeljawad, T.: Fractional difference operators with discrete generalized Mittag-Leffler kernels. Chaos Solitons Fractals 126, 315-324 (2019)

14. Abdeljawad, T.: On delta and nabla Caputo fractional differences and dual identities. Discrete Dyn. Nat. Soc. 2013, Article ID 406910 (2013)

15. Abdeljawad, T.: Dual identities in fractional difference calculus within Riemann. Adv. Differ. Equ. 2013(1), 36 (2013)

16. Jarad, F., Abdeljawad, T., Baleanu, D., Bicen, K.: On the stability of some discrete fractional nonautonomous systems. Abstr. Appl. Anal. 2012, Article ID 476581 (2012)

17. Goodrich, C., Peterson, A.C.: Discrete Fractional Calculus. Springer, Berlin (2015)

18. Khennaoui, A.-A., Ouannas, A., Bendoukha, S., Grassi, G., Wang, X., Pham, V.-T.: Generalized and inverse generalized synchronization of fractional-order discrete-time chaotic systems with non-identical dimensions. Adv. Differ. Equ. 2018(1), 1 (2018)

19. Khennaoui, A.-A., Ouannas, A., Bendoukha, S., Wang, X., Pham, V.-T.: On chaos in the fractional-order discrete-time unified system and its control synchronization. Entropy 20(7), 530 (2018)

20. Wu, G.-C., Baleanu, D.: Discrete fractional logistic map and its chaos. Nonlinear Dyn. 75(1-2), $283-287$ (2014)

21. Wu, G.-C., Baleanu, D., Xie, H.-P., Chen, F.-L.: Chaos synchronization of fractional chaotic maps based on the stability condition. Phys. A, Stat. Mech. Appl. 460, 374-383 (2016)

22. Huang, L.-L., Baleanu, D., Wu, G.-C., Zeng, S.-D.: A new application of the fractional logistic map. Rom. J. Phys. 61(7-8), 1172-1179 (2016)

23. Kassim, S., Hamiche, H., Djennoune, S., Bettayeb, M.: A novel secure image transmission scheme based on synchronization of fractional-order discrete-time hyperchaotic systems. Nonlinear Dyn. 88(4), 2473-2489 (2017)

24. Lian, K.-Y., Chiang, T.-S., Liu, P.: Discrete-time chaotic systems: applications in secure communications. Int. J. Bifurc. Chaos 10(09), 2193-2206 (2000)

25. Feki, M., Robert, B., Gelle, G., Colas, M.: Secure digital communication using discrete-time chaos synchronization. Chaos Solitons Fractals 18(4), 881-890 (2003)

26. Jun-Guo, L., Yu-Geng, X.: Chaos communication based on synchronization of discrete-time chaotic systems. Chin. Phys. 14(2), 274 (2005)

27. Stork, M.: Digital chaotic systems examples and application for data transmission. In: 2009 International Conference on Electrical and Electronics Engineering-ELECO 2009, pp. 78. IEEE (2009)

28. Kocarev, L., Szczepanski, J., Amigó, J.M., Tomovski, I.: Discrete chaos-i: theory. IEEE Trans. Circuits Syst. I, Regul. Pap. 53(6), 1300-1309 (2006)

29. Lozi, R.: Un attracteur étrange du type attracteur de Hénon. J. Phys., Colloq. 39(C5), 5-9 (1978)

30. Hénon, M.: A two-dimensional mapping with a strange attractor. In: The Theory of Chaotic Attractors, pp. 94-102. Springer, Berlin (1976)

31. Hitzl, D.L., Zele, F.: An exploration of the Hénon quadratic map. Phys. D, Nonlinear Phenom. 14(3), $305-326$ (1985)

32. Stefański, K.: Modelling chaos and hyperchaos with 3-d maps. Chaos Solitons Fractals 9(1-2), 83-93 (1998)

33. Baier, G., Sahle, S.: Design of hyperchaotic flows. Phys. Rev. E 51(4), 2712 (1995)

34. Itoh, M., Yang, T., Chua, L.O.: Conditions for impulsive synchronization of chaotic and hyperchaotic systems. Int. J. Bifurc. Chaos 11(02), 551-560 (2001)

35. Pecora, L.M., Carroll, T.L.: Synchronization in chaotic systems. Phys. Rev. Lett. 64(8), 821 (1990)

36. Wang, X.: Chaos in Complex Nonlinear Systems Publishing House of Electronics Industry. House of Electronics Industry, Beijing (2003)

37. Khennaoui, A.-A., Ouannas, A., Bendoukha, S., Grassi, G., Wang, X., Pham, V.-T., Alsaadi, F.E.: Chaos, control, and synchronization in some fractional-order difference equations. Adv. Differ. Equ. 2019(1), 1 (2019) 
38. Abdeljawad, T., Banerjee, S., Wu, G.-C.: Discrete tempered fractional calculus for new chaotic systems with short memory and image encryption. Optik 218, 163698 (2020)

39. Wu, G.-C., Luo, M., Huang, L.-L., Banerjee, S.: Short memory fractional differential equations for new memristor and neural network design. Nonlinear Dyn. 100, 3611-3623 (2020)

40. Wu, G.-C., Deng, Z.-G., Baleanu, D., Zeng, D.-Q.: New variable-order fractional chaotic systems for fast image encryption. Chaos, Interdiscip. J. Nonlinear Sci. 29(8), 083103 (2019)

41. Boccaletti, S., Grebogi, C., Lai, Y.-C., Mancini, H., Maza, D.: The control of chaos: theory and applications. Phys. Rep. 329(3), 103-197 (2000)

42. Fradkov, A.L., Evans, R.J., Andrievsky, B.R.: Control of chaos: methods and applications in mechanics. Philos. Trans. R. Soc. A, Math. Phys. Eng. Sci. 364(1846), 2279-2307 (2006)

43. Ouannas, A., Khennaoui, A.-A., Bendoukha, S., Grassi, G.: On the dynamics and control of a fractional form of the discrete double scroll. Int. J. Bifurc. Chaos 29(06), 1950078 (2019)

44. Ouannas, A., Khennaoui, A.-A., Grassi, G., Bendoukha, S.: On chaos in the fractional-order Grassi-Miller map and its control. J. Comput. Appl. Math. 358, 293-305 (2019)

45. Khennaoui, A.A., Ouannas, A., Boulaaras, S., Pham, V.-T., Taher Azar, A.: A fractional map with hidden attractors: chaos and control. Eur. Phys. J. Spec. Top. 229, 1083-1093 (2020)

46. Ouannas, A., Bendoukha, S., Khennaoui, A.-A., Grassi, G., Wang, X., Pham, V.-T.: Chaos synchronization of fractional-order discrete-time systems with different dimensions using two scaling matrices. Open Phys. 17(1), 942-949 (2019)

47. Cermák, J., Gyori, I., Nechvátal, L.: On explicit stability conditions for a linear fractional difference system. Fract. Calc. Appl. Anal. 18(3), 651 (2015)

48. Baleanu, D., Wu, G.-C., Bai, Y.-R., Chen, F.-L.: Stability analysis of Caputo-like discrete fractional systems. Commun. Nonlinear Sci. Numer. Simul. 48, 520-530 (2017)

49. Wu, G.-C., Baleanu, D., Luo, W.-H.: Lyapunov functions for Riemann-Liouville-like fractional difference equations. Appl. Math. Comput. 314, 228-236 (2017)

50. Pawluszewicz, E.: Constrained controllability of the-difference fractional control systems with Caputo type operator. Discrete Dyn. Nat. Soc. 2015, Article ID 638420 (2015)

51. Karanjkar, D., Chatterji, S., Venkateswaran, P.: Trends in fractional order controllers. Int. J. Emerg. Technol. Adv. Eng. 2(3), 383-389 (2012)

52. Mozyrska, D., Pawłuszewicz, E., Wyrwas, M.: The $h$-difference approach to controllability and observability of fractional linear systems with Caputo-type operator. Asian J. Control 17(4), 1163-1173 (2015)

53. Mozyrska, D., Pawluszewicz, E.: Controllability of $h$-difference linear control systems with two fractional orders. In: Proceedings of the 13th International Carpathian Control Conference (ICCC), pp. 501-506. IEEE (2012)

54. Pawłuszewicz, E., Mozyrska, D.: Remarks on observability of $h$-difference linear control systems with two fractional orders. In: Proceedings of the 14th International Carpathian Control Conference (ICCC), pp. 292-296. IEEE (2013)

55. Khennaoui, A.-A., Ouannas, A., Bendoukha, S., Grassi, G., Lozi, R.P., Pham, V.-T.: On fractional-order discrete-time systems: chaos, stabilization and synchronization. Chaos Solitons Fractals 119, 150-162 (2019)

56. Li, Y., Sun, C., Ling, H., Lu, A., Liu, Y.: Oligopolies price game in fractional order system. Chaos Solitons Fractals 132, $109583(2020)$

\section{Submit your manuscript to a SpringerOpen ${ }^{\circ}$ journal and benefit from:}

- Convenient online submission

- Rigorous peer review

- Open access: articles freely available online

- High visibility within the field

- Retaining the copyright to your article

Submit your next manuscript at $\gg$ springeropen.com 